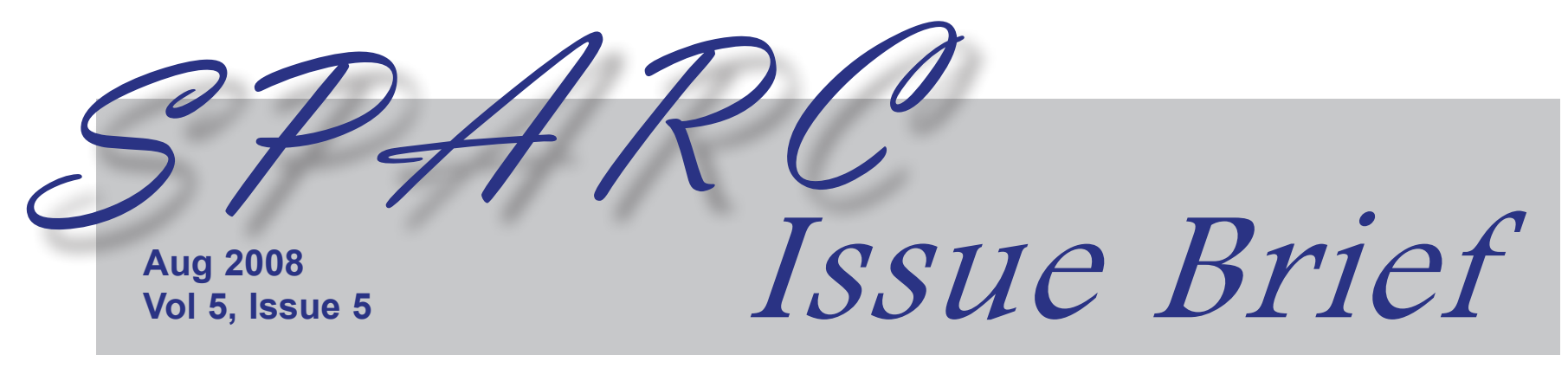

\title{
Institutional Review Boards: What do they do? How do they protect subjects?
}

\section{Christopher Jackson, MA, Philip Candilis, MD, Suzanne Garverich, BA, Lauren Gellar, MS, CHES, Charles Lidz, PhD, Teresa Roach, MA}

\begin{abstract}
A lthough the history of medical research is full of glorious achievements, there are also horror stories: the Nazi medical experiments, the Tuskegee study, and others. In response to these problems, the federal government created the concept of an Institutional Review Board to oversee the ethics of research. IRBs consist of at least one committee of clinical and research experts and at least one member from outside the institution (the Board) and a staff of varying sizes that oversees the administrative aspects of research ethics review. Given the widespread reliance on Institutional Review Boards (IRBs) as oversight bodies for assuring ethical conduct of research, it is surprising that there has been little if any investigation of their decision-making process.
\end{abstract}

IRBs regulate all types of research, including research that is done on people with mental illness. For this reason, a team at SPARC has undertaken a general descriptive study of IRB practices funded by a grant from the National Institutes of Health (NIH). This research examines the IRB practices in 10 of the 25 largest medical research facilities in the United States. We are focusing on decision-making about newly submitted research study applications that are presented to biomedical IRBs. By applying both quantitative and qualitative research techniques to tape-recorded IRB deliberations, and semi-structured interviews with IRB staff and reviewers, we will: 1) interpret the content of IRB decisions about individual research protocols, 2) characterize the interaction processes by which IRBs make decisions and 3) describe the IRB decision-making processes in terms of organizational decision-making theory. Although we are particularly interested in IRB oversight of research about people with mental illness, this study

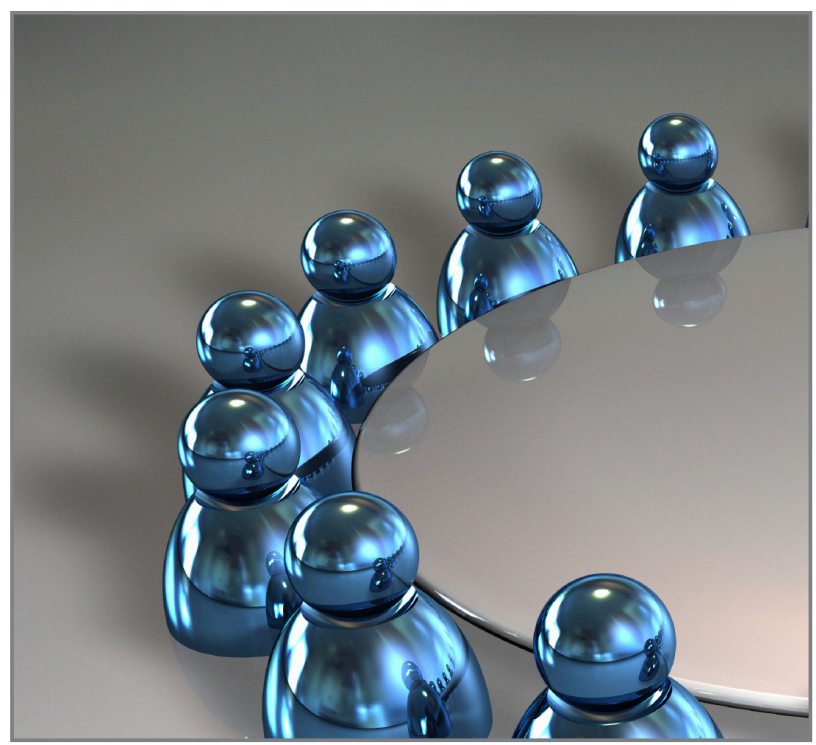

looks at all medical research so as to appreciate the broad context of IRB work.

\section{Methods}

We have collected data from seven of an anticipated 10 IRBs in the form of qualitative interviews, observed and audio recorded panel meetings and redacted research study applications. We have observed a total of 14 biomedical IRB panels (two panels per institution) and conducted approximately 70 personal interviews with IRB panelists and staff members. With the collaboration of IRB staff, applications on the agenda for the two observed meetings are identified and redacted of all identifying information. The study team then reviews the applications in preparation for interviewing the panelists assigned as reviewers for the IRB meeting. In the days following the panel meeting, the reviewer(s) of each application are interviewed about their individual 
roles on the IRB, the structure and functionality of the board, their experiences reviewing protocols and their issues and concerns about the particular application that they reviewed. The interview elicits their perspectives about the study applications, isolates points of interest and asks questions that are used to clarify the organization and functioning of the board. Interview questions are designed to uncover the various roles and expectations that structure the group decision-making processes. Interviews typically last forty-five minutes to one hour, are digitally recorded and are conducted immediately following the meeting date at a location most convenient for the participant.

\section{Preliminary Findings}

One of the initial steps in IRB decision-making is problem identification. Specifically, what do the staff and committee members find to be a concern or a problem with an application or a consent form. A major focus of our research has become determining the cultural/ cognitive resources that are used to make these judgments. Each type of problem requires applying different resources of knowledge. Some types of expertise employed by IRBs include:

- Medical--will the subject receive less than what is standard care; is the experimental intervention safe;

- Methods--adequate statistical power;

- Ethical--assessing the weight of benefits to society at large;

- Psychological--how will people with cognitive and/or emotional problems be affected;

- Cultural--how will a consent form be interpreted by ethnic minorities or international subjects.

Our research has revealed that the role expectations and job responsibilities of IRB panelists and IRB staff members vary across institutions. For example, a task such as reviewing an application for completeness may be performed by an IRB staff member at one site but may be performed by an IRB panel member at another. IRB staff and panelists use a variety of techniques and devices to facilitate their processing and review of study documents: including checklists, readability scales, established rules, and precedents, as well as rule books and guidelines. Such techniques may increase the reliability of IRB reviews. Some sites increase efficiency of reviews by having staff do some tasks prior to the meeting, thus reducing meeting time. For example, some IRBs assign a staff member the task of making consent forms readable by using readability scales as opposed to taking up valuable and more expensive panel meeting time to do this.

While the roles and responsibilities of community members (i.e., members of the IRB who are drawn from the larger community and not employees of the medical center) on IRBs vary in some degree across sites, based on personal interviews collected, most panelists agree that nonmedical secondary reviewers should focus their critiques on the consent forms and other non-medical/technical issues. Rarely do the community members believe they are qualified to review the appropriateness of medical procedures or details of pharmacology. These findings suggest that lay members of IRBs should be encouraged to see their role as a specialized one. IRB chairs and physicians alike commented on their appreciation of lay members' opinions and cited them as a useful balance to the medical perspective. Additionally, non-medical members sometimes act as patient advocates.1,2

One issue that has been a matter of dispute, both in the wider literature and among our interviewees, is the IRB's role in review of scientific methodology. Some reviewers stated that it was not the IRBs role to assess scientific methodologies and some even expressed doubts about their ability to do so. Others felt that a methodologically flawed design would not produce valid results. They saw this as an important subject protection issue because subjects would be put through a potentially risky process for no useful purpose. Clearly there are variations in reviewers' perceptions of their role in assessing scientific methodology and additional clarification of the IRBs role in this matter is necessary.

\section{Future Research}

This study focuses on the structure and practices of biomedical IRBs primarily at academic medical centers. Future research of a similar nature should be undertaken examining psychosocial, specialty and review boards designed exclusively to review applications involving vulnerable populations such as children, people with mental illness and prisoners. Since there is growing use of private "for profit" review boards for multi-site studies, it would be valuable to look at the structure and practices of private review boards. Given the growth of IRB's resources and responsibilities, additional research on IRBs is important to all potential subjects, including people diagnosed with mental illness.

\section{References}

1. Ghio, J. (1980). What is the role of a public member on an IRB? IRB: A Review of Human Subjects Research, 2(2), 7.

2. Porter, J. (1987). How unaffiliated/nonscientist members of institutional review boards see their roles. IRB: A Review of Human Subjects Research, 9(6), 1-6.

Visit us on-line at www.umassmed.edu/sparc Send Comments tosparc@umassmed.edu 